\title{
Multidisciplinary evaluation and management of obstructive hypertrophic cardiomyopathy in 2020: Towards the HCM Heart Team
}

\author{
Francesco Pelliccia ${ }^{\mathrm{a}, *}$, Ottavio Alfieri ${ }^{\mathrm{b}}$, Paolo Calabrò ${ }^{\mathrm{c}, \mathrm{d}}$, Franco Cecchi ${ }^{\mathrm{e}}$, Paolo Ferrazzi ${ }^{\mathrm{f}}$, Felice Gragnano ${ }^{\mathrm{c}, \mathrm{d}}$, \\ Juan Pablo Kaski ${ }^{g}$, Giuseppe Limongelli ${ }^{\mathrm{d}, \mathrm{h}}$, Martin Maron ${ }^{\mathrm{i}}$, Claudio Rapezzi ${ }^{\mathrm{j}}$, Hubert Seggewiss ${ }^{\mathrm{k}}$, \\ Magdi H. Yacoub ${ }^{1}$, Iacopo Olivotto ${ }^{\mathrm{m}}$
}

a Department of Cardiovascular Sciences, University Sapienza of Rome, Rome, Italy

b Department of Cardiovascular and Thoracic Surgery, San Raffaele University Hospital, Milan, Italy

' Division of Cardiology, A.O.R.N. 'Sant'Anna e San Sebastiano', Caserta, Italy

' Department of Translational Medical Sciences, University of Campania 'Luigi Vanvitelli', Naples, Italy

e IRCCS Istituto Auxologico Italiano, Department of Cardiovascular, Neural and Metabolic Sciences, San Luca Hospital, Milan, Italy

${ }^{\mathrm{f}}$ Hypertrophic Cardiomyopathy Center, Policlinico di Monza, Monza, Italy

g Centre for Inherited Cardiovascular Diseases, Great Ormond Street Hospital, University College London Institute of Cardiovascular Science, London, UK

${ }^{\text {h }}$ Institute of Cardiovascular Sciences, University College of London, St. Bartholomew's Hospital, London, UK

${ }^{i}$ Hypertrophic Cardiomyopathy Center, Division of Cardiology, Tufts Medical Center, Boston, MA, USA

j Cardiology, University of Ferrara \&' Maria Cecilia Hospital, GVM Care and Research, Cotignola, Italy

${ }^{\mathrm{k}}$ Klinikum Würzburg Mitte - Standort Juliusspital, Würzburg, Germany

${ }^{1}$ Heart Science Centre, National Heart and Lung Institute, Imperial College London, London, UK

${ }^{\mathrm{m}}$ Cardiomyopathy Unit, Careggi University Hospital, Florence, Italy

\section{A R T I C L E I N F O}

\section{Article history:}

Received 5 January 2020

Accepted 10 January 2020

Available online $\mathrm{xxxx}$

\section{Keywords:}

Alcohol septal ablation

Hypertrophic cardiomyopathy

Gradient

Left ventricular outflow tract

Myectomy

Obstruction

\begin{abstract}
A B S T R A C T
Patients with hypertrophic cardiomyopathy (HCM) exhibit a variable phenotype with ventricular hypertrophy as the cardinal manifestation and left ventricular (LV) outflow tract obstruction (LVOTO) as a key pathophysiologic determinant. Patients with severe LVOTO usually present with exertional dyspnea, exertional syncope, and heart failure symptoms, while successful relief of LVOTO by pharmacological or invasive interventions leads to a dramatic improvement in clinical status. Proper management of obstructive HCM remains challenging and poses numerous clinical dilemmas. Since the development of surgical myectomy over half a century ago, progress in the management of LVOTO in HCM has paralleled technological advances in genetic testing, cardiac imaging, arrhythmic prophylaxis, cardiac surgery and interventional cardiology. These changes have been incorporated in dedicated scientific guidelines on both sides of the Atlantic. However, either the 2011 American guidelines or the 2014 European guidelines remain largely based on expert consensus for lack of recommendations with level of evidence A regarding any of the treatment options commonly employed in HCM. Consequently, management of obstructive HCM patients remains largely subjective and dependent on clinical judgment, local expertise, and patient preference. Following the trend that has emerged for other cardiac diseases amenable to invasive interventions, adequate evaluation and management of obstruction in HCM today requires a multidisciplinary team capable of optimizing referral, choosing the best available options, minimizing complications and ensuring state-of-the-art results. The concept of an HCM Heart Team is coming of age. This review aims to provide an update of available pharmacologic and invasive options for the management of LVOTO in HCM, either in adulthood or in childhood, highlighting areas for multidisciplinary integration and future development.
\end{abstract}

(c) 2020 Elsevier B.V. All rights reserved.

\section{Preamble: the clinical burden of obstruction in HCM}

Hypertrophic cardiomyopathy (HCM) is a complex cardiovascular disorder characterized by unexplained non dilated left ventricular

* Corresponding author at: Department of Cardiovascular Sciences, University Sapienza of Rome, Via del Policlinico 155, 00161 Rome, Italy.

E-mail address: f.pelliccia@mclink.it (F. Pelliccia).
(LV) thickening in the absence of other cardiac or systemic diseases justifying the degree of hypertrophy observed [1]. The morphological, histological, and clinical phenotypes of HCM reflect complex interactions among a large number of pathophysiologic players triggered by the causal genetic mutation, generally affecting sarcomere protein genes. Patients with HCM exhibit a variable phenotype with ventricular hypertrophy as the cardinal manifestation, myocyte hypertrophy, disarray, microvascular remodeling and myocardial fibrosis as key pathological 
hallmarks, and impaired ventricular filling and dynamic LV outflow tract (LVOT) gradients as key pathophysiologic features [1].

LVOT obstruction (LVOTO) in HCM is caused by the systolic anterior movement (SAM) of elongated mitral valve leaflets, contacting the septum at the subaortic level [2]. Patients with severe LVOTO usually present with exertional dyspnea or angina, and may experience severe functional limitation. Relief of obstruction by pharmacological or invasive interventions usually leads to a dramatic improvement in their clinical status and often affords complete relief of limiting symptoms [3].

Because of the heterogeneous manifestations, natural history and prognosis, tailored management of obstructive HCM remains challenging and poses numerous clinical dilemmas. Since the development of surgical myectomy over half a century ago [4], progress in the management of LVOTO in HCM has paralleled technological advances in genetic testing, cardiac imaging, arrhythmic prophylaxis, cardiac surgery and interventional cardiology. These changes have been incorporated in dedicated scientific guidelines for the diagnosis and treatment of the disease on both sides of the Atlantic. However, neither the 2011American Heart Association/American College of Cardiology guidelines [5] nor the 2014 European Society of Cardiology guidelines [6] could provide any recommendation with a level of evidence A for treatment options commonly employed in HCM. Current recommendations are based on a bulk of well-designed but retrospective studies as well as on expert opinions. Consequently, management of LVOTO varies largely based on local expertise and patient preference. However, adequate evaluation and management of obstruction in HCM today requires a multidisciplinary team capable of optimizing referral, choosing the best available options, minimizing complications and ensuring stateof-the-art results. Following the trend that has emerged for other cardiac diseases amenable to invasive interventions, the concept of a HCM Heart Team is coming of age. This review aims to provide an update of available pharmacologic and invasive options for the management of LVOTO in HCM, either in adulthood or in childhood, highlighting areas for multidisciplinary integration and future development.

\section{Pathophysiology and evaluation of obstruction in HCM}

LVOTO has been the source of periodic controversies and paradigm shifts concerning its genesis and prognostic impact for over 50 years [2] (Fig. 1). The pathophysiology of HCM was first described in the '60s by Braunwald et al. [7] and Wigle et al. [8] who labeled the disease as idiopathic hypertrophic subaortic stenosis and muscular subaortic stenosis, emphasizing the obstructive nature of the disease. In the preechocardiographic era, the presence of an LVOT gradient was considered a sine qua non of the disease. Following the introduction of Doppler echocardiography, the true prevalence and clinical spectrum of HCM were progressively defined: HCM is now known to occur in at least 1:500 of the general population, with about a third manifesting obstruction in resting conditions and another third only with exercise. Typically, the LVOT gradient varies with loading conditions and contractility due to its dynamic nature. Indeed, the gradient can be provoked or augmented by multiple factors, including sympathomimetic agents, premature ectopic beats, or Valsalva. Thus, exercise echocardiography frequently unmasks latent LVOTO [9]. Therefore, most HCM patients have some degree of impedance at the LVOT either at rest or with provocation.

LVOTO results from the interplay of multiple mechanisms. The key anatomic determinant is the hypertrophic basal anteroseptal wall, coupled with a small-sized ventricular cavity narrowing the LVOT. The functional determinant of obstruction is mitral SAM and the consequent mitral-septal contact [3]. Initially considered pathognomonic of HCM, SAM is now recognized in other pathologic conditions altering the geometry and function of the LV and mitral valve. In HCM, however, SAM is particularly common due to the primary abnormalities of the mitral valve (enlargement and elongation) and subvalvular apparatus

\section{LVOT gradient and obstruction: Controversies and paradigm shifts}

1960 The gradient exists but it is not synonymous with obstruction since it is caused by the precipitous high velocity flow within a conduit or by ventricular catheter entrapment

The dynamic gradient is indeed related to a LVOT 'obstruction' but it's a late phenomenon occurring when more than $2 / 3$ of stroke volume has been ejected, so that functional and clinical consequences are limited

Obstructive forms represent a minority (around one third of patients)

1980 The aspiration of the anterior leaflet of the mitral valve toward interventricular septum within a tight LVOT due to Venturi effect is the main cause of obstruction

Septal reduction (surgery or ablation) is effective in reducing LVOT gradient and improving symptoms

Exercise can unmask LVOT gradient in more than $50 \%$ of cases, so that $\mathrm{HCM}$ is predominantly an obstructive disease

1998 LVOT obstruction is the cause of heart failure in many patients and is a risk factor of sudden cardiac death

The mechanism of LVOT obstruction is multifactorial and includes primary abnormalities of the mitral apparatus and abnormal forces either pushing and aspiring the anterior leaflet of the mitral valve against the septum

2020 Mitral valve remodeling (associated with myectomy) is an essential component of surgical treatment of LVOT obstruction

Fig. 1. LVOT gradient and obstruction: 50 years of controversies and paradigm shifts. $\mathrm{HCM}=$ hypertrophic cardiomyopathy; LVOT = left ventricular outflow tract.

(papillary muscle hypertrophy and displacement, abnormal chordal attachments). In addition, evidence now exists that altered flow vectors generated in the LV cavity along with changes in outflow tract geometry favor the contact between the anterior leaflet of the mitral valve and the hypertrophic septum [10]. These synergistic mechanisms push the leaflets into the outflow tract ('drag forces') resulting in LVOTO and loss of leaflet coaptation, the latter resulting in functional, posteriorly directed mitral regurgitation [11]. SAM is considered severe if there is septal contact for $>30 \%$ of the duration of systole.

In a minority of patients ( $1 \%)$, impedance to flow occurs at midventricular level and is unrelated to SAM. Mid-ventricular obstructive HCM is characterized by the presence of a pressure gradient between the apical and basal portion of the LV, almost creating separate chambers, as a is the result of systolic apposition of the septum and LV free wall, with the interposition of an anteriorly displaced papillary muscle. Mid-ventricular obstruction may be associated with an LV apical aneurysm, in the absence of epicardial coronary artery disease.

Doppler echocardiography is the first-line imaging technique to diagnose obstructive HCM [5,6], as it documents SAM and allows evaluation of LV morphology and repeated, real-time measures of LVOT gradients at rest and with Valsalva, thus permitting the hemodynamic classification of any HCM patient [10] (Supplemental Fig. 1). Although patients may generate large gradients under non-physiologic or pharmacological provocation, exercise (stress) echocardiography is considered the only reliable tool for documenting the presence of latent LVOTO in physiological conditions [5,6] (Supplemental Table 1). Cardiac catheterization is rarely required today, unless discordance between symptoms and non-invasive tests occurs [6]. Besides being a relevant determinant of outcome [12] (Supplemental Fig. 2), LVOTO is a major cause of symptoms and should be routinely sought during clinical evaluation. North American guidelines advise exercise stress echocardiography for identification of latent obstruction in the absence of a hemodynamically significant $(\geq 50 \mathrm{mmHg}$ ) LVOT gradient at rest or with Valsalva [5]. Conversely, European recommendations warrant exercise echocardiography in case of symptoms attributable to LVOTO (e.g. dyspnea, chest pain, exercise limitation, and/or impaired 
consciousness). Testing for latent LVOTO is not considered necessary in asymptomatic patients unless "relevant to lifestyle advice and decisions on medical treatment" [6].

\section{Medical management including novel pharmacologic options}

Pharmacological treatment represents the first approach to patients with obstructive HCM [13] and may be effective in controlling LVOT gradients and symptoms. Both North American and European guidelines have provided specific recommendations [5,6] (Table 1). Beta-blockers (e.g. atenolol, nadolol, bisoprolol, and metoprolol) are the most popular and effective agents employed and may be titrated based on symptoms, heart rate response, and blood pressure. Non-dihydropyridine calcium channel blockers such as verapamil and diltiazem are considered less effective, although they can be used in patients who are intolerant or have contraindications to beta-blockers. Disopyramide, a class IA antiarrhythmic agent, can be used in association with beta-blockers or calcium channel blockers to improve symptoms and reduce gradients in patients with LVOTO by virtue of its negative inotropic effect. Whereas beta-blockers are most effective on provocable LVOTO, disopyramide is the most effective agent on resting obstruction. Side effects, such as QT prolongation and its anticholinergic properties, can limit its use and impair compliance. Diuretics may be used in patients with pulmonary congestion and should be prescribed at minimal effective doses. Careful observation is required to avoid hypovolemia, hypotension and intensification or provocation of LVOTO. In patients with obstructive HCM and concomitant hypertension or coronary artery disease requiring pharmacological treatment, caution is required with vasodilators and/or positive inotropic agents and vasodilators (e.g. phosphor-diesterase inhibitors) because of the risk of exacerbation of LVOTO [14,15].

Recently, a novel therapeutic approach has emerged based on the hypothesis that HCM is triggered by a hyper-dynamic state directly caused by disease-causing sarcomere mutations on the contractile apparatus $[16,17]$. By selectively decreasing the affinity of myosin for actin, downstream consequences of sarcomere mutations might be countered in HCM patients, including prevention of phenotype development in the early stages of the disease. A small-molecule allosteric myosin inhibitor, mavacamten (MYK-461; Myokardia, San Francisco, CA, US), has been recently developed to restore physiologic contractility and energetic balance in HCM by decreasing the adenosine triphosphatase activity of the cardiac myosin heavy chain. After promising results obtained in animal models, mavacamten has been tested in HCM patients in the PIONEER-HCM study (A Phase 2 Open-label Pilot Study Evaluating MYK-461 in Subjects With Symptomatic Hypertrophic Cardiomyopathy and Left Ventricular Outflow Tract Obstruction) [18]. The study enrolled 21 patients aged 18 to 70 years. The primary endpoint was the change in post-exercise peak LVOT gradient at week 12 as compared to baseline. Patients enrolled in the PIONEER-HCM were

Table 1

Pharmacologic indications to treat symptoms associated with HCM based on the 2011 American and 2014 European guidelines.

\begin{tabular}{|c|c|c|}
\hline Drug & 2011 ACC/AHA guidelines [5] & 2014 ESC guidelines [6] \\
\hline Beta-blockers & I - Level of evidence B & I - Level of evidence B \\
\hline Calcium blockers & $\begin{array}{l}\text { I - Level of evidence B } \\
\text { Ilb - Level of evidence C }\end{array}$ & $\begin{array}{l}\text { I - Level of evidence B } \\
\text { Ila - Level of evidence C }\end{array}$ \\
\hline $\begin{array}{l}\text { - Verapamil } \\
\text { - Diltiazem }\end{array}$ & & \\
\hline Disopyramide & $\begin{array}{l}\text { Ila - Level of evidence B } \\
\text { Ila - Level of evidence B }\end{array}$ & $\begin{array}{l}\text { I - Level of evidence B } \\
\text { IIb - Level of evidence C }\end{array}$ \\
\hline $\begin{array}{l}\text { - Alone } \\
\text { - In association }\end{array}$ & & \\
\hline Diuretics & IIb - Level of evidence C & IIb - Level of evidence C \\
\hline
\end{tabular}

$\mathrm{ACC}=$ American College of Cardiology; $\mathrm{AHA}=$ American Heart Association; ESC = European Society of Cardiology. divided into higher dose and lower dose cohorts. In the higher dose cohort including 11 patients, mavacamten significantly reduced postexercise peak LVOT gradient from $125 \mathrm{mmHg}$ to $19 \mathrm{mmHg}$ ( $p=$ 0.002 ) and improved peak VO2 from $20.7 \mathrm{ml} / \mathrm{kg} / \mathrm{min}$ to $24.6 \mathrm{ml} / \mathrm{kg} /$ $\min (p=0.004)$ at week 12 . Of note, LVOT gradient reduction was paralleled by a significant reduction in resting LV ejection fraction (mean change: $-15 \%[\mathrm{CI},-23 \%$ to $-6 \%]$ ). Plans are currently underway for the pivotal EXPLORER-HCM (Clinical Study to Evaluate Mavacamten in Adults With Symptomatic Obstructive Hypertrophic Cardiomyopathy) study, which would enroll 250 patients and evaluate changes in peak VO2 and subjective improvement in symptoms based on NYHA classification as the primary endpoints [19]. Another negative myosin modulator (CK-274; Cytokinetics, US) is also currently under clinical development for HCM patients with LVOTO [20].

\section{Invasive septal reduction strategies}

\subsection{Surgical myectomy}

Septal reduction therapy should be considered in patients with an LVOTO gradient greater or equal to $50 \mathrm{mmHg}$, moderate to severe symptoms and/or exertional syncope despite maximally tolerated drug therapy. There are currently two main strategies for septal reduction: surgical myectomy and alcohol septal ablation (ASA). Surgical myectomy involves the removal of a small amount (5-10 g) of hypertrophic tissue from the basal inter-ventricular septum. Since the first myectomy was performed in the late 1950s [21], constant developments and variations have been applied to the procedure. Septal resection is now often extended circumferentially and distally, to the level of the papillary muscles, to avoid residual mid-ventricular obstruction and to ensure laminar flow through the LVOT area. Unfortunately, extreme heterogeneity of septal morphology and LV geometry can make septal myectomy extremely challenging. Careful pre-operative assessment by means of novel imaging tools (e.g. cardiac magnetic resonance) now allows tailoring of the septal excision on an individual basis, including apical extension [22]. Indeed, cardiac magnetic resonance provides reliable assessment of the location, pattern and distribution of LV wall thickening in the basal septum aiding pre-operative planning and improving surgical outcome [22]. When the obstruction is localized distally, at the mid-ventricular level, the transaortic approach may prove challenging. In order to avoid residual obstruction after surgery due to inadequate length of septal excision, an original application of cryoenergy can improve the transaortic exposure of the interventricular septum and thus enable surgeons to perform very distal myectomies [23]. Alternatively, a combined transaortic and transapical approach may be used in expert hands. The surgical intervention also allows the possibility to address concomitant valvular and subvalvular abnormalities in a personalized manner, including mobilization of the right and/or left fibrous trigones, thus enhancing the dynamic behaviour of the LVOT [24], and leaflet excision/retraction to reduce mismatch. Furthermore, there are specific modalities to intervene in cases characterized by severe obstruction in the presence of minimal septal hypertrophy (maximum thickness $\leq 16 \mathrm{~mm}$ ) including chordal cutting [25], the edge-toedge technique or, in the presence of structural valve disease, prosthetic valve replacement. When concomitant papillary muscle abnormalities are present, dissection and reduction of the anomalous papillary apparatus along with chordal cutting and even plication of the mitral valve are performed as part of the contemporary myectomy operation in order to completely eliminate LVOT obstruction (Fig. 2). Surgery is the technique of choice for LVOTO due to congenital discrete subaortic stenosis and in HCM patients with coexistant aortic stenosis [24]. Finally, concomitant ablation for atrial fibrillation by means of Cox-Maze procedure can be performed at time of septal myectomy. With this approach, an excellent outcome of freedom from atrial fibrillation episodes during long-term follow-up has been reported [26]. 

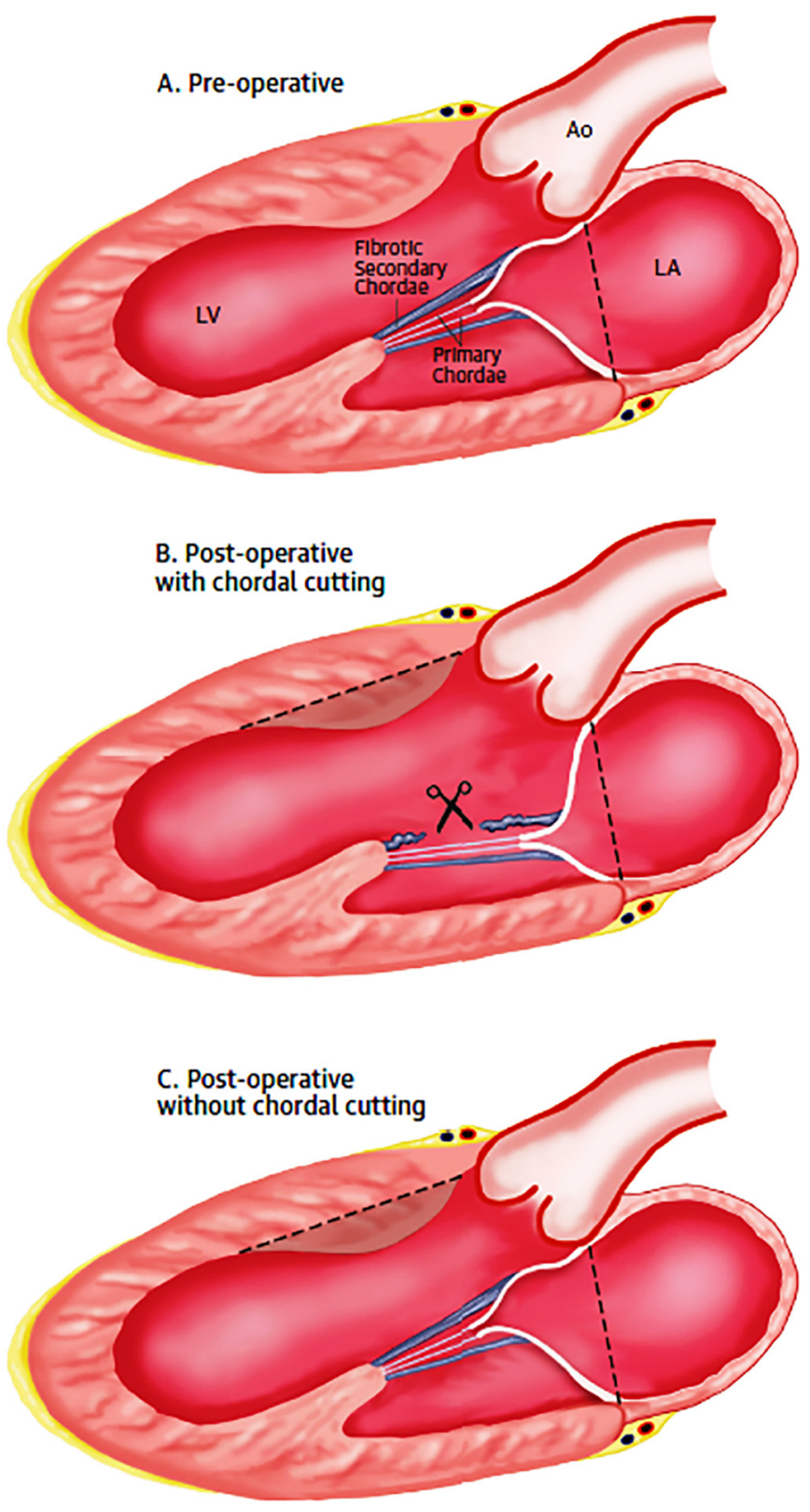

Fig. 2. Secondary chordal cutting in obstructive HCM: effects of secondary chordal cutting on the geometry and function of the mitral valve apparatus. (A) In patients with obstructive hypertrophic cardiomyopathy, fibrotic and retracted mitral valve secondary chordae contribute to displace the body of the anterior leaflet into the left ventricular outflow tract. (B) Cutting selected abnormal chordae (in combination with a shallow septal myectomy) moves the mitral valve apparatus and leaflet coaptation point away from the outflow tract to a more posterior and normal position in the left ventricular cavity, substantially increasing outflow tract size and decreasing mitral valve tenting area. (C) Isolated septal myectomy (i.e., without associated chordal cutting) does not alter the anterior displacement of the mitral valve apparatus. Ao = aorta; $\mathrm{LA}=$ left atrium; $\mathrm{LV}=$ left ventricle. The figure is from Ferrazzi P et al. and with permission [25].

\subsection{Alcohol septal ablation}

Three decades after the first myectomy, Sigwart performed the first non-surgical septal reduction in HCM by percutaneous intracoronary alcohol injection [27]. ASA aims to induce a limited iatrogenic infarction localized at the basal septum, positioned to coincide with the point of contact of the anterior mitral valve leaflet during SAM. Local remodeling and thinning of the septum are effective in abolishing LVOTO. It is a catheter-based technique that involves the injection of a small amount of desiccated alcohol (1.5-2.5 mL) into a septal perforator [28]. The evaluation of LVOT geometry, septum morphology, and valve apparatus as well as coronary anatomy is crucial to predict ASA feasibility. Major structural anomalies of the LVOT, mitral valve and papillary muscles must be excluded, as these will prevent an optimal results. The anatomy of coronary circulation is carefully assessed by coronary angiography, and the correct identification of one or more 'target' septal branches is of paramount importance [27] (Fig. 3). As a rule, the first septal perforator is initially tested as target vessel for ASA. Following proximal occlusion of the septal branch by balloon to avoid spillover, contrast echocardiography is used to confirm that the branch supplies the basal septum including the area of mitral contact. If there is clear mismatch with such area, or if there is evidence of contrast diffusion to other areas (e.g. distal septum or right ventricle), another branch is chosen. Alcohol is injected only when contrast echocardiography suggests optimal placement.

\subsection{Patient selection}

Selection criteria for ASA are generally based on anatomical features and increased operative risk advising against surgery. However, the selection process is controversial and largely depends on local expertise [28] (Supplemental Table 2).No randomized trial has adequately compared the long term effects of ASA versus myectomy and existing comparisons are largely based on registries, case studies or meta-analysis of observational studies. Such a trial appears unfeasible: based on early trials comparing coronary angioplasty versus by-pass, Olivotto et al. provided an estimate that 34,000 consecutive patients with HCM would need to be screened, in order to reach the required total of 1200 enrolled patients (with 600 patients in each group) achieving statistical power [29].

Based on available evidence, the 2011 North American guidelines consider septal myectomy as the gold standard technique for septal reduction, and advise against performing ASA in the presence of young age, severe septal thickness ( $>25-30 \mathrm{~mm}$ ), mid-ventricular obstruction and concomitant cardiac disease [5]. ASA is recommended in the elderly, in patients with significant comorbidities increasing surgical risk, or when patients refuse open-heart surgery [5]. European guidelines do not give priority to one technique over another, but suggest an individual assessment by an experienced multidisciplinary team [6]

Numerous studies now exist showing that both surgical myectomy and ASA provide durable relief of symptoms and reduction of LVOTO [28] with excellent long-term survival (Supplemental Fig. 3). To date, several meta-analyses have found ASA to be non-inferior to myectomy in terms of subsequent functional improvement, short-term and longterm mortality [30,31]. Most importantly, procedure-related mortality in experienced centers for both techniques myectomy and ASA approximates $1 \%[30,31]$. Based on such degree of confidence, HCM patients with Class II symptoms are increasingly referred to expert centers for earlier septal reduction therapy, particularly when young or in the presence of high resting LVOT gradients, severe left atrial dilatation and/or pulmonary hypertension.

Despite the excellent results obtained by referral centers, several caveats should be considered when considering septal reduction therapy in HCM patients. First and foremost, operative outcomes vary dramatically based on regional experience: low-flow centers may have unacceptable mortality rates, as well as suboptimal long-term results. The importance of choosing the appropriate team and technique cannot be overstated. In addition, several technical pitfalls must be considered. Muscular septal excision with myectomy may damage the conduction system, leading to a left bundle branch block. Thus, patients with preexistent right bundle branch should either receive ASA or should be informed of a high risk of requiring a pacemaker post-operatively [32]. However, pacemaker rates can be almost two times higher in those undergoing ASA than those undergoing myectomy. Indeed, ASA can cause a right bundle branch block in up to $50-60 \%$ of patients, and it is best 

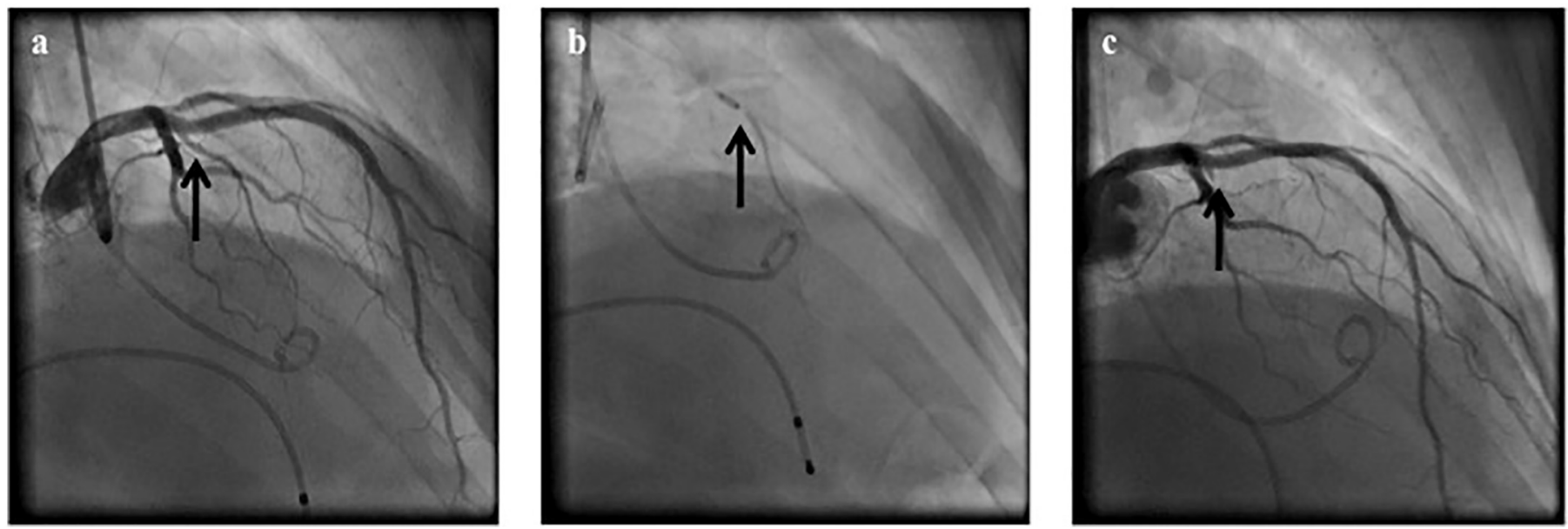

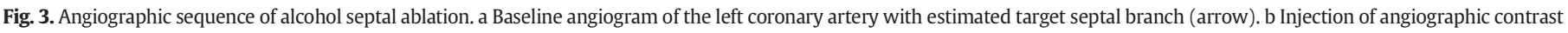

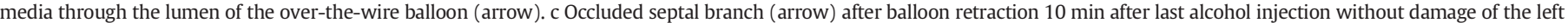
anterior descending artery.

avoided in patients with preexistent left bundle branch block [32]. Finally, concerns persist regarding the very long-term structural and arrhythmogenic potential of the scar generated by ASA, a major reason why the procedure is usually avoided in children and adolescents. However, an increased arrhythmic propensity following ASA has not been observed in the recent literature [32].

In a proportion of patients who have received invasive septal reduction, LVOTO can recur during follow-up, particularly in patients treated with ASA. Similar to staged coronary angioplasty, repeat ASA can be easily performed at the price of an increase in the risk of pacemaker implantation, although surgery is also very effective. A repeat myectomy is rare in expert hands and is generally due to incomplete elimination of LVOTO by the initial procedure, often due to failure to extend the resection apically towards the mid-ventricle $[21,22]$.

In conclusion, both ASA and myectomy have shown excellent results in experienced hands. However, most patients will not be equally poised for both procedures when assessed by an experienced clinician. Pre-operative evaluation is key in selecting the ideal patient for ASA versus surgical myectomy. In this regard, the formidable possibilities of contemporary imaging allow tailored pre-operative planning and are key in optimizing results while reducing complications. The two procedures require extensive knowledge of the disease, and the learning curve is demanding. Evidence now exists that available expertise may prove more important than theoretical considerations in choosing the right technique.

\section{Approaching obstruction in children with HCM}

HCM is a rare condition in childhood, with an estimated prevalence of 2.8/100,000, and an annual incidence of between 0.24 and 0.47 per 100,000 [33]. Although most cases are caused by autosomal dominantly-inherited sarcomere protein gene mutations [33], compared to adults, the etiology of HCM in childhood is heterogeneous and includes malformation syndromes (e.g. Noonan syndrome and related disorders of the RAS-MAPK pathway); inborn errors of metabolism (e.g. Pompe disease and other storage disorders); mitochondrial cytopathies; and neuromuscular disorders (e.g. Friedreich ataxia) [34]. Children with inborn errors of metabolism and malformation syndromes in particular frequently present in infancy (below 1 year of age) with signs and symptoms of congestive cardiac failure caused by biventricular outflow tract obstruction and have a substantially poorer prognosis than those presenting later with sarcomeric or nonsyndromic disease [35].
LVOTO is present at rest in approximately one quarter of children with HCM [35], and can cause symptoms of dyspnoea, chest pain, syncope and presyncope, resulting from an acute reduction in cardiac output, myocardial ischemia and elevated LV filling pressures. Echocardiographic evaluation should include not just documentation of the gradient at rest and with provocation manoeuvres (e.g. Valsalva) but also an assessment of the mechanism of obstruction. In children with RASopathy syndromes in particular, there is frequently a polyvalvulopathy that can result in mitral valve dysplasia and accessory attachments from the papillary muscles to the base of the ventricular septum and/or the underside of the anterior mitral valve leaflet, contributing to the presence of complex LVOTO and mitral regurgitation [36]. Exercise stress echocardiography has also been shown to be feasible in children with HCM in the assessment of provocable LVOTO [37], which may be present in up to $50-60 \%$ of symptomatic individuals without a resting LVOT gradient.

The management of LVOTO in children is primarily directed at relieving symptoms, and treatment of asymptomatic children with obstructive HCM remains controversial. While there are no prospective data on the long-term effects of treatment of LVOTO in children, some retrospective studies have suggested a survival benefit with pharmacological and/or surgical therapies [38]. In some centers, treatment of resting LVOT gradients $>75-90 \mathrm{mmHg}$ regardless of symptoms is recommended [34].

Beta-blockers are the initial drug of choice in the treatment of symptomatic LVOTO in children with HCM. The agents most commonly used include propranolol, atenolol, bisoprolol and metoprolol titrated to symptoms [34]. When symptoms persist despite adequate betablockade, the addition of disopyramide (dose $6-20 \mathrm{mg} / \mathrm{kg} / \mathrm{day}$ ) can result in a reduction on the LVOT gradient and improvement in symptoms [39]. Calcium channel blockers such as verapamil and diltiazem can also be considered as alternative or additional agents and result in improved LV relaxation and symptomatic improvement in obstructive HCM; they should be initiated with caution, usually in an in-patient setting, in children with resting LVOTO, as they can sometimes precipitate acute hemodynamic decompensation [34].

For children with obstructive HCM who remain symptomatic despite maximal pharmacological therapy, or where there is evidence of a fixed obstructive component (e.g. due to accessory mitral valve chordal attachments or discrete subaortic membrane with significant contribution to the LVOT gradient), surgical myectomy can result in significant symptomatic improvement together with reduction in degree of mitral regurgitation and left atrial size [38]. In expert centers, complication rates (including atrioventricular block, iatrogenic ventricular 
septal defect and damage to the mitral or aortic valves) are low and mortality rates generally $<2 \%$ [38].

Dual chamber pacing has been reported to improve symptoms in children with HCM and LVOTO, but faster heart rates and shorter atrio-ventricular conduction velocities compared to adults make optimal programming problematic [40]. In addition, lead and devicerelated complications (including infective endocarditis) are relatively common in the paediatric age group [40]. Therefore, pacemaker treatment for symptomatic LVOTO in children with HCM is limited to those individuals who are undergoing implantation of an implantable cardioverter-defibrillator or in the very rare cases where a myectomy is not possible. Other interventional procedures, such as alcohol septal ablation, or radiofrequency ablation of the ventricular septum [41], are currently experimental and should not be used outside the research setting.

Historical observational studies have suggested that surgical myectomy may improve survival and reduce the risk of sudden death in children with obstructive HCM [38], but there are no prospective, controlled data to confirm this. A recent meta-analysis [42] identified 6 studies assessing LVOTO as a potential risk factor for mortality in children with HCM ( 2 for sudden cardiac death and 4 for all-cause cardiovascular death) but only one reported a significant association. Furthermore, two recent retrospective studies evaluating sudden cardiac death risk in children and adolescents with HCM suggested an inverse relationship between sudden death risk and left ventricular outflow tract gradient $[43,44]$ The role of LVOTO in risk stratification in childhood HCM, therefore, remains uncertain and requires further investigation.

\section{The need for a personalized approach and the 'HCM Heart Team'}

The choice of septal reduction therapy should always be based on individual patient's characteristics, following a multifactorial approach. ASA remains controversial in children and young adults because of the lack of long-term data [45]. Moreover, this strategy may be less effective in patients with severe hypertrophy and/or extensive septal scar, due to the intrinsic limitation of alcohol, and troublesome in case of mild septal hypertrophy, due to the risk of septal perforation. The ultimate decision for the strategy to adopt should always take into account patient preference [6]. Thereby, a shared decision-making approach is always to be preferred, discussing risks and benefits of each treatment strategy, matching clinical and procedural aspects with the needs and preferences of the individual patient. For this purpose, it is crucial that the decisional process is carried out with a multi-disciplinary approach by an experienced team working in a dedicated cardiomyopathy center of excellence. The concept of 'heart team', ideally including clinical and interventional cardiologists, and cardiac surgeons has shown to improve discussion and decision-making between interventional versus surgical approach in both coronary artery and valvular heart disease. Following the same formula, in HCM patients management, a 'Cardiomyopathy Team' should be excellent in analyzing and interpreting diagnostic tests and contextualizing them in the clinical status of the patient, critically appraising the need for a septal reduction intervention, and the individual benefit-risk ratio with either ASA or myectomy [46]. This team should be at the least be composed by a clinical cardiologist, an interventional cardiologist and a cardiac surgeon with recognized experience in HCM. Implementing the strategical role of cardiomyopathy teams working in experienced centers is a critical step in the management of this population, as the results of both percutaneous and surgical septal reduction strategy are largely dependent on the experience of the operators/institutions [47]. In the case of myectomy, alarming data suggest that the real-world mortality rate for the procedure approximates 4-16\% [47]. Similarly, in the case of for ASA best results are proven to be achieved only by highly-experienced operators [48]. Accordingly, the American guidelines for HCM strictly recommend that all invasive SRT procedures should be performed by experienced operators in the context of a comprehensive HCM clinical program, aiming at minimizing the rate of death and major complications at $<1 \%$ and $<3 \%$ respectively for isolated septal myectomy [5].

\section{Future perspectives}

Pharmacological options in patients with HCM have not evolved in the last decades from the basic objectives of relief of symptoms and improvement in functional capacity. Currently, two myosin inhibitors are being developed for clinical use in symptomatic obstructive HCM with a Phase 3 randomized controlled trial. To date, however, no drug therapy has yet been shown to prolong survival or reduce the risk of sudden cardiac death. The development of novel pharmacological strategies that are capable of altering the natural history of this disease has been recognized by the National Heart Lung and Blood Institute as an unmet need and a research priority [49].

To date, judicious use of the available pharmacological armamentarium may already provide sufficient control of the most common clinical manifestations and complications related to LVOTO, allowing normal longevity in the majority of male and female patients with HCM [50]. Serial assessment and early identification of disease progression are key for a timely implementation of available therapies. As regards interventional techniques, both surgical myectomy and ASA techniques will continue to exist and improve. Optimizing efficacy while reducing pacemaker dependency and improving long-term survival is the obvious ultimate goal. The two procedures should be viewed as complementary rather than competing treatments, enriching the therapeutic armamentarium for HCM patients.

HCM is transitioning into an exciting phase of a pharmacologic discovery and increasing availability of safe and effective invasive intervention for LVOTO. While many of these therapies hold significant promise to constantly improve the standards of treatment for HCM patients, significant gaps in knowledge remain in order to radically address the molecular basis and natural history of the disease. This target should set a common agenda for physicians and basic scientist alike.

Supplementary data to this article can be found online at https://doi. org/10.1016/j.ijcard.2020.01.021.

\section{Declaration of competing interest}

The authors declare that they have no known competing financial interests or personal relationships that could have appeared to influence the work reported in this paper.

\section{References}

[1] A.J. Marian, E. Braunwald, Hypertrophic cardiomyopathy: genetics, pathogenesis, clinical manifestations, diagnosis, and therapy, Circ. Res. 121 (2017) 749-770.

[2] B.J. Maron, M.S. Maron, E.D. Wigle, E. Braunwald, The 50-year history, controversy, and clinical implications of left ventricular outflow tract obstruction in hypertrophic cardiomyopathy: from idiopathic hypertrophic subaortic stenosis to hypertrophic cardiomyopathy, J. Am. Coll. Cardiol. 54 (2009) 181-200.

[3] B.J. Maron, Clinical course and management of hypertrophic cardiomyopathy, $\mathrm{N}$ Engl. J. Med. 379 (2018) 655-668.

[4] D. Teare, Asymmetrical hypertrophy of the heart in young adults, Br. Heart J. 20 (1958) 1-8.

[5] B. J. Gersh, B.J. Maron, R.O. Bonow, et al. 2011 ACCF/AHA guideline for the diagnosis and treatment of hypertrophic cardiomyopathy: a report of the American College of Cardiology Foundation/American Heart Association task force on practice guidelines developed in collaboration with the American Association for Thoracic Surgery, American Society of Echocardiography, American Society of Nuclear Cardiology, Heart Failure Society of America, Heart Rhythm Society, Society for Cardiovascular Angiography and Interventions, and Society of Thoracic Surgeons. J. Am. Coll. Cardiol. 58 (2011) e212-e260.

[6] P.M. Elliott, A. Anastasakis, M.A. Borger, et al., 2014 ESC guidelines on diagnosis and management of hypertrophic cardiomyopathy: the task force for the diagnosis and management of hypertrophic cardiomyopathy of the European Society of Cardiology, Eur. Heart J. 35 (2014) 2733-2779.

[7] E. Braunwald, H.N. Oldham Jr., J. Ross Jr., J.W. Linhart, D.T. Mason, L. Fort III, The circulatory response of patients with idiopathic hypertrophic subaortic stenosis: to nitroglycerin and to the Valsalva maneuver, Circulation 29 (1964) 422-431. 
[8] E.D. Wigle, R.O. Heimbecker, R.W. Gunton, Idiopathic ventricular septal hypertrophy causing muscular subaortic stenosis, Circulation 26 (1962) 325-340.

[9] M.S. Maron, I. Olivotto, A.G. Zenovich, et al., Hypertrophic cardiomyopathy is predominantly a disease of left ventricular outflow tract obstruction, Circulation 114 (2006) 2232-2239.

[10] D. Lu, B. Hailesealassie, I. Ventoulis, et al., Impact of peak provoked left ventricular outflow tract gradients on clinical outcomes in hypertrophic cardiomyopathy, Int. J. Cardiol. 243 (2017) 290-295.

[11] L.M. Charls, Systolic anterior motion of the anterior mitral valve leaflet post-surgical mitral valve repair, Heart. Lung. 32 (2003) 402-406.

[12] F. Pelliccia, V. Pasceri, G. Limongelli, et al. Long-term outcome of nonobstructive versus obstructive hypertrophic cardiomyopathy: a systematic review and metaanalysis. Int. J. Cardiol. 243 (2017) 379-384.

[13] E. Ammirati, R. Contri, R. Coppini, F. Cecchi, M. Frigerio, I. Olivotto, Pharmacological treatment of hypertrophic cardiomyopathy: current practice and novel perspectives, Eur. J. Heart. Fail. 18 (2016) 1106-1118.

[14] T. Wilder, D.M. Ryba, D.F. Wieczorek, B.M. Wolska, R.J. Solaro, N-acetylcysteine reverses diastolic dysfunction and hypertrophy in familial hypertrophic cardiomyopathy, Am. J. Physiol. Heart. Circ. Physiol. 309 (2015) H1720-H1730.

[15] Axelsson, K. Iversen, N. Vejlstrup, et al. Efficacy and safety of the angiotensin II receptor blocker losartan for hypertrophic cardiomyopathy: the INHERIT randomised double-blind, placebo-controlled trial. Lancet. Diabetes, Endocrinol. 3 (2015) 123-131.

[16] Olivotto, J.L. Hellawell, R. Farzaneh-Far, et al. Novel approach targeting the complex pathophysiology of hypertrophic cardiomyopathy: the impact of late sodium current inhibition on exercise capacity in subjects with symptomatic hypertrophic cardiomyopathy (LIBERTY-HCM) Trial. Circ. Heart. Fail. 9 (2016) e002764.

[17] F. Pelliccia, V. Pasceri, G. Marazzi, G. Rosano, C. Greco, C. Gaudio, A pilot randomized study of ranolazine for reduction of myocardial damage during elective percutaneous coronary intervention, Am. Heart J. 163 (2012) 1019-1023.

[18] D. Heitner, D. Jacoby, S.J. Lester, Mavacamten treatment for obstructive hypertrophic cardiomyopathy: a clinical trial, Ann. Intern. Med. 170 (2019) 741-748.

[19] Olivotto, C. Ho, D. Jacoby, et al. EXPLORER-HCM: phase 3 randomized, multi-center, double-blind, placebo-controlled study to evaluate mavacamten (MYK-461) in adults with symptomatic obstructive hypertrophic cardiomyopathy. Circulation. 138 (2018) A16466.

[20] L.A. Robertson, D.R. Armas, E. Robbie, et al. A first in human study of the selective cardiac myosin inhibitor, CK-3773274. J. Cardiac. Fail. 25 (2019) S79-S80.

[21] A.G. Morrow, C. Lambrew, E. Braunwald, Idiopathic hypertrophic subaortic stenosis: II. Operative treatment and the results of pre- and postoperative hemodynamic evaluations, Circulation 29 (1964) 120-151.

[22] P. Spirito, I. Binaco, D. Poggio, et al., Role of preoperative cardiovascular magnetic resonance in planning ventricular septal myectomy in patients with obstructive hypertrophic cardiomyopathy, Am. J. Cardiol. 123 (2019) 1517-1526.

[23] Pozzoli, L. Vicentini, S. Thelin, E. Lapenna, L. Nilsson, O. Alfieri, Application of cryoenergy to improve septal exposure during transaortic septal myectomy in hypertrophic obstructive cardiomyopathy, Gen. Thorac. Cardiovasc. Surg. 66 (2018) 243-245.

[24] M.H. Yacoub, A. Afifi, H. Saad, H. Aguib, A. ElGuindy, Current state of the art and future of myectomy, Ann. Cardiothorac. Surg. 6 (2017) 307-317.

[25] P. Ferrazzi, P. Spirito, A. Iacovoni, et al., Transaortic chordal cutting: mitral valve repair for obstructive hypertrophic cardiomyopathy with mild septal hypertrophy, J. Am. Coll. Cardiol. 66 (2015) 1687-1696.

[26] G. Boll, E.J. Rowin, B.J. Maron, W. Wang, H. Rastegar, M. Maron, Efficacy of combined Cox Maze IV and ventricular septal myectomy for treatment of atrial fibrillation in patients with obstructive hypertrophic cardiomyopathy, Am. J. Cardiol. 125 (2020) $120-126$.

[27] L. Faber, H. Seggewiss, U. Gleichmann, Percutaneous transluminal septal myocardial ablation in hypertrophic obstructive cardiomyopathy: results with respect to intraprocedural myocardial contrast echocardiography, Circulation. 98 (1998) 2415-2421, https://doi.org/10.1161/01.cir.98.22.2415.

[28] F. Pelliccia, G. Niccoli, F. Gragnano, et al., Alcohol septal ablation for hypertrophic obstructive cardiomyopathy: a contemporary reappraisal, EuroIntervention 15 (2019) 411-417.
[29] Olivotto, S.R. Ommen, M.S. Maron, F. Cecchi, B.J. Maron, Surgical myectomy versus alcohol septal ablation for obstructive hypertrophic cardiomyopathy. Will there ever be a randomized trial, J. Am. Coll.Cardiol. 50 (2007) 831-834.

[30] M. Liebregts. A systematic review and meta-analysis of long-term outcomes after septal reduction therapy in patients with hypertrophic cardiomyopathy. JACC. Heart. Failure. 3 (2015) 896-905.

[31] K. Singh, M. Qutub, K. Carson, B. Hibbert, C. Glover, A meta-analysis of current status of alcohol septal ablation and surgical myectomy for obstructive hypertrophic cardiomyopathy, Catheter. Cardiovasc. Interv. 88 (2016) 107-115.

[32] Batzner, B. Pfeiffer, A. Neugebauer, D. Aicha, C. Blank, H. Seggewiss, Survival after alcohol septal ablation in patients with hypertrophic obstructive cardiomyopathy, J. Am. Coll. Cardiol. 72 (2018) 3087-3094.

[33] J.P. Kaski, P. Syrris, M.T. Esteban, et al., Prevalence of sarcomere protein gene mutations in preadolescent children with hypertrophic cardiomyopathy, Circ. Cardiovasc. Genet. 2 (2009) 436-441.

[34] J.P. Moak, J.P. Kaski, Hypertrophic cardiomyopathy in children, Heart 98 (2012) 1044-1054.

[35] G. Norrish, E. Field, K. McLeod, et al., Clinical presentation and survival of childhood hypertrophic cardiomyopathy: a retrospective study in United Kingdom, Eur. Heart J. 40 (2019) 986-993.

[36] G. Calcagni, G. Limongelli, A. D'Ambrosio, et al., Cardiac defects, morbidity and mortality in patients affected by RASopathies. CARNET study results, Int. J. Cardiol. 245 (2017) 92-98

[37] P. Ciliberti, I. McLeod, F. Cairello, et al., Semi-supine exercise stress echocardiography in children and adolescents: feasibility and safety, Pediatr. Cardiol. 36 (2015) 633-639.

[38] K. Minakata, J.A. Dearani, P.W. O'Leary, G.K. Danielson, Septal myectomy for obstructive hypertrophic cardiomyopathy in pediatric patients: early and late results, Ann. Thorac. Surg. 80 (2005) 1424-1429.

[39] M.J. O'Connor, K. Miller, R.E. Shaddy, et al., Disopyramide use in infants and children with hypertrophic cardiomyopathy, Cardiol. Young 28 (2018) 530-535.

[40] R.J. Czosek, K. Meganathan, J.B. Anderson, T.K. Knilans, B.S. Marino, P.C. Heaton, Cardiac rhythm devices in the pediatric population: utilization and complications, Heart Rhythm. 9 (2012) 199-208.

[41] N. Sreeram, M. Emmel, J.V. de Giovanni, Percutaneous radiofrequency septal reduction for hypertrophic obstructive cardiomyopathy in children, J. Am. Coll. Cardiol. 58 (2011) 2501-2510.

[42] G. Norrish, N. Cantarutti, E. Pissaridou, et al. Risk factors for sudden cardiac death in childhood hypertrophic cardiomyopathy: a systematic review and meta-analysis. Eur. J. Prev. Cardiol. 24 (2017) 1220-1230.

[43] S. Balaji, M.P. DiLorenzo, F.A. Fish, et al. Risk factors for lethal arrhythmic events in children and adolescents with hypertrophic cardiomyopathy and an implantable defibrillator: an international multicenter study. Heart. Rhythm. 16 (2019) $1462-1467$.

[44] G. Norrish, T. Ding, E. Field, et al. Development of a novel risk prediction model for sudden cardiac death in childhood hypertrophic cardiomyopathy (HCM RiskKids). JAMA. Cardiol. 2019 Aug 14. doi: https://doi.org/10.1001/jamacardio.2019. 2861.

[45] M. Liebregts, P.A. Vriesendorp, J.M. ten Berg, Alcohol septal ablation for obstructive hypertrophic cardiomyopathy: a word of endorsement, J. Am. Coll. Cardiol. 70 (2017) 481-488.

[46] N. Ad, L.M. Wei, It is the time for a heart team approach for patients with hypertrophic cardiomyopathy, J. Thorac. Cardiovasc. Surg. 154 (2017) e105-e106.

[47] S.R. Ommen, R.A. Nishimura, Hypertrophic cardiomyopathy-one case per year?: a clarion call to do what is right, JAMA Cardiol. 1 (2016) 333-334.

[48] L.K. Kim, R.V. Swaminathan, P. Looser, et al., Hospital volume outcomes after septal myectomy and alcohol septal ablation for treatment of obstructive hypertrophic cardiomyopathy: US nationwide inpatient database, 2003-2011, JAMA. Cardiol. 1 (2016) 324-332.

[49] T. Force, R.O. Bonow, S.R. Houser, et al., Research priorities in hypertrophic cardiomyopathy: report of a Working Group of the National Heart, Lung, and Blood Institute, Circulation 122 (2010) 1130-1133.

[50] F. Pelliccia, G. Limongelli, C. Autore, J.R. Gimeno-Blanes, C. Basso, P. Elliott, Sexrelated differences in cardiomyopathies, Int. J. Cardiol. 286 (2019) 239-243. 